\title{
Computing Differentially Rotating Neutron Stars Obeying Realistic Equations of State by Using Hartle's Perturbation Method
}

\author{
Anastasios Katelouzos, Vassilis Geroyannis \\ Department of Physics, University of Patras, Patras, Greece \\ Email: ktasos@physics.upatras.gr,vgeroyan@upatras.gr
}

Received June 12, 2013; revised July 14, 2013; accepted July 23, 2013

Copyright (C) 2013 Anastasios Katelouzos, Vassilis Geroyannis. This is an open access article distributed under the Creative Commons Attribution License, which permits unrestricted use, distribution, and reproduction in any medium, provided the original work is properly cited.

\begin{abstract}
In this paper, we use the well-known Hartle's perturbation method in order to compute models of differentially rotating neutron stars obeying realistic equations of state. In our numerical treatment, we keep terms up to third order in the angular velocity. We present indicative numerical results for models satisfying a particular differential rotation law. We emphasize on computing the change in mass owing to this differential rotation law.
\end{abstract}

Keywords: Change in Mass; Differential Rotation; General-Relativistic Models; Neutron Stars; Numerical Methods; Realistic Equations of State

\section{Introduction}

In [1] we have implemented the well-known Hartle's perturbation method ([2-4]), by keeping terms up to third order in the angular velocity, to the computation of differentially rotating neutron stars simulated by generalrelativistic polytropic models (angular momentum, moment of inertia, rotational kinetic energy, and gravitational potential energy are quantities drastically corrected by the third-order approach). The present study is continuation of [1] regarding models of differentially rotating neutron stars obeying realistic "equations of state" (EOS, EOSs).

For clarity and convenience, we adopt here the definitions and symbols used in [1].

The motivation for computing models of differentially rotating neutron stars obeying realistic equations of state by implementing Hartle's pertrurbation method arises from the fact that, to the extent of the knowledge of the authors, respective results seem to be "missing" from the bibliography; and this seems to be true even for the alternative (iterative) methods, except for certain computations regarding maximum mass models as in [5]. Thus, interested readers may have the opportunity to take into account our results or to compare with respective results of their own numerical methods.

\section{Preliminaries}

Theory and computations are extensively presented in [1] (Sections 2-5). The Schwarchild metric of a nonrotating spherical object, expressed in spherical coordinates $(r, \theta, \varphi)$ is defined as in [2] (Equation (25)),

$$
\mathrm{d} s^{2}=-\mathrm{e}^{v} \mathrm{~d} t^{2}+\mathrm{e}^{\lambda} \mathrm{d} r^{2}+r^{2}\left(\mathrm{~d} \theta^{2}+\sin ^{2} \theta \mathrm{d} \phi^{2}\right) .
$$

The nonrotating model obeys the relativistic Oppenheimer-Volkoff equations of: (1) the hydrostatic equilibrium ([2], Equation (28)),

$$
\frac{\mathrm{d} P}{\mathrm{~d} r}=-\frac{(\rho+P)\left(m+4 \pi r^{3} P\right)}{r(r-2 m)},
$$

and (2) the mass-energy ([2], Equation (29a)),

$$
\frac{\mathrm{d} m}{\mathrm{~d} r}=4 \pi r^{2} \rho .
$$

The EOSs used in the computations of this study are given in Table 1. In general, for densities up to $10^{4} \mathrm{~g} \cdot \mathrm{cm}^{-3}$, the EOSs are joined onto the Feynman-Metropolis-Teller EOS [11]. For densities in the interval $\left[1 \times 10^{4} \mathrm{~g} \cdot \mathrm{cm}^{-3}, 4.3 \times 10^{11} \mathrm{~g} \cdot \mathrm{cm}^{-3}\right]$ they join onto the Baym-Pethick-Sutherland EOS [12]. For densities above neutron drip density (i.e. $\sim 4 \times 10^{11} \mathrm{~g} \cdot \mathrm{cm}^{-3}$ ) and typically in the range $\left[4.3 \times 10^{11} \mathrm{~g} \cdot \mathrm{cm}^{-3}, 2 \times 10^{14} \mathrm{~g} \cdot \mathrm{cm}^{-3}\right]$, they 
join onto the Baym-Bethe-Pethick EOS [13] (for a detailed discussion on realistic EOSs, see e.g. [14], Section $3.1)$.

\section{Results and Discussion}

As said above, the computations of the present study proceed as in [1] (Section 5). We shall not repeat here such details; interested readers can find the full numerical treatment there.

We present numerical results for the EOSs AU, C, F, FPS, and G. All models computed here have central density $\rho_{\mathrm{c}}=1 \times 10^{15} \mathrm{gcm}^{-3}$. When resolved in the state of uniform rotation ([1], Section 6, Equation (209)), all models are assumed to be rotated with their Keplerian angular velocity $\Omega_{\mathrm{K}}$. Several methods have been developed for the computation of $\Omega_{\mathrm{K}}$ (for a general discussion on such methods, see e.g. [14], Section 3.7). In this study, in order to compute $\Omega_{\mathrm{K}}$ for our models, we use the well-known RNS package [15].

When resolved in the state of differential rotation ([1], Section 6, Equation (210)), all models have angular velocity at the equatorial surface, $\Omega_{\mathrm{e}}$, equal to their Keplerian angular velocity,

$$
\Omega_{\mathrm{e}}=\Omega_{\mathrm{K}} .
$$

The differential rotation function on the equatorial plane $\delta \Omega(r, \theta=\pi / 2)$ ([1], Section 4.1, Equation (63)) increases from zero at the equatorial surface up to a maximum at the center, which (maximum) depends on the dimensionless model parameter $\hat{\mathcal{A}}^{-1}$ ([1], Section
4.1, Equation (74)); the value $\hat{\mathcal{A}}^{-1}=0$ describes a uniform rotation, while its gradually increasing values 0.3 , 0.5 , and 0.7 (studied in this work) yield differential rotations with respectively increasing maxima $\delta \Omega(r=0, \theta=\pi / 2)$. Accordingly, the central angular velocity $\Omega_{\mathrm{c}}$ becomes equal to

$$
\Omega_{c}=\Omega_{K}+\delta \Omega(r=0, \theta=\pi / 2)
$$

and the ratio $\Omega_{\mathrm{c}} / \Omega_{\mathrm{e}}$ is written as

$$
\Omega_{\mathrm{c}} / \Omega_{\mathrm{e}}=1+\delta \Omega(r=0, \theta=\pi / 2) .
$$

Tables 2-6 give numerical results for certain significant quantities of our models. In each table, there are two groups of quantities: The first group, labeled "corrections due to differential rotation", contains the changes occured in the three kinds of mass due to differential rotation. The second group, labeled "total quantities and significant ratios", presents total values of certain significant quantities and, also, values of some interesting ratios. Emphasizing on the increase in the baryonic mass

Table 1. Realistic EOSs used in the computations.

\begin{tabular}{clc}
\hline EOS & Description & Reference \\
\hline C & Modified Reid soft core potential & {$[6]$} \\
F & Modified Reid potential & {$[7]$} \\
G & Modified Reid soft core potential & {$[8]$} \\
AU & AV14 + UVII; Wiringa, Fiks and Fabrocini & {$[9]$} \\
FPS & UV14 + TNI; Lorenz, Ravenhall and Pethick & {$[10]$} \\
\hline
\end{tabular}

Table 2. Numerical results given in cgs for the EOS AU; differential rotation for three values of the model parameter $\hat{\mathcal{A}}^{-1}$.

\begin{tabular}{llll}
\hline Model parameter, $\hat{\mathcal{A}}^{-1}$ & \multicolumn{1}{c}{0.3} & 0.5 & 0.7 \\
\hline Corrections due to differential rotation & & & \\
Gravitational mass, $\delta M_{\mathrm{DR}}$ & $6.201(+31)$ & $1.851(+32)$ & $4.007(+32)$ \\
Baryonic mass, $\delta M_{\mathrm{BDR}}$ & $9.167(+31)$ & $2.286(+32)$ & $4.677(+32)$ \\
Proper mass, $\delta M_{\mathrm{PDR}}$ & $9.659(+31)$ & $2.420(+32)$ & $4.966(+32)$ \\
Total quantities and significant ratios & & & $1.780(+00)$ \\
Angular velocity ratio, $\Omega_{\mathrm{c}} / \Omega_{\mathrm{c}}$ & $1.143(+00)$ & $1.398(+00)$ & $2.808(+33)$ \\
Gravitational mass, $M_{\mathrm{tot}}$ & $2.469(+33)$ & $2.592(+33)$ & $3.085(+33)$ \\
Baryonic mass, $M_{\mathrm{Btot}}$ & $2.709(+33)$ & $2.846(+33)$ & $3.679(-01)$ \\
Ratio $\left(\delta M_{\mathrm{B}}+\delta M_{\mathrm{BDR}}\right) / M_{\mathrm{B}}$ & $2.011(-01)$ & $2.619(-01)$ & $3.232(+33)$ \\
Proper mass, $M_{\mathrm{P} \text { tot }}$ & $2.832(+33)$ & $2.977(+33)$ & $1.054(+49)$ \\
Angular momentum, $J_{\text {tot }}$ & $8.600(+48)$ & $9.376(+48)$ & $5.331(+52)$ \\
Rotational kinetic energy, $T_{\text {tot }}$ & $3.450(+52)$ & $4.135(+52)$ & $4.343(+53)$ \\
Gravitational potential energy, $|W|_{\text {tot }}$ & $3.604(+53)$ & $3.873(+53)$ & $1.227(-01)$ \\
Ratio $(T /|W|)_{\text {tot }}$ & $9.572(-02)$ & $1.068(-01)$ & \\
\hline
\end{tabular}


Table 3. Numerical results given in cgs for the EOS C; differential rotation for three values of the model parameter $\hat{\mathcal{A}}^{-1}$.

\begin{tabular}{|c|c|c|c|}
\hline Model parameter, $\hat{\mathcal{A}}^{-1}$ & 0.3 & 0.5 & 0.7 \\
\hline \multicolumn{4}{|l|}{ Corrections due to differential rotation } \\
\hline Gravitational mass, $\delta M_{\mathrm{DR}}$ & $6.879(+31)$ & $2.041(+32)$ & $4.385(+32)$ \\
\hline Baryonic mass, $\delta M_{\mathrm{BDR}}$ & $1.069(+32)$ & $2.573(+32)$ & $5.179(+32)$ \\
\hline Proper mass, $\delta M_{\mathrm{PDR}}$ & $1.146(+32)$ & $2.776(+32)$ & $5.600(+32)$ \\
\hline \multicolumn{4}{|l|}{ Total quantities and significant ratios } \\
\hline Angular velocity ratio, $\Omega_{\mathrm{c}} / \Omega_{\mathrm{c}}$ & $1.150(+00)$ & $1.416(+00)$ & $1.816(+00)$ \\
\hline Gravitational mass, $M_{\text {tot }}$ & $3.063(+33)$ & $3.199(+33)$ & $3.433(+33)$ \\
\hline Baryonic mass, $M_{\text {Btot }}$ & $3.369(+33)$ & $3.520(+33)$ & $3.780(+33)$ \\
\hline Ratio $\left(\delta M_{\mathrm{B}}+\delta M_{\mathrm{BDR}}\right) / M_{\mathrm{B}}$ & $1.735(-01)$ & $2.259(-01)$ & $3.166(-01)$ \\
\hline Proper mass, $M_{\text {Ptot }}$ & $3.574(+33)$ & $3.737(+33)$ & $4.019(+33)$ \\
\hline Angular momentum, $J_{\text {tot }}$ & $1.266(+49)$ & $1.391(+49)$ & $1.577(+49)$ \\
\hline Rotational kinetic energy, $T_{\text {tot }}$ & $4.574(+52)$ & $5.567(+52)$ & $7.314(+52)$ \\
\hline Gravitational potential energy, $|W|_{\text {tot }}$ & $5.042(+53)$ & $5.390(+53)$ & $5.996(+53)$ \\
\hline Ratio $(T /|W|)_{\text {tot }}$ & $9.071(-02)$ & $1.033(-01)$ & $1.220(-01)$ \\
\hline
\end{tabular}

Table 4. Numerical results given in cgs for the EOS F; differential rotation for three values of the model parameter $\hat{\mathcal{A}}^{-1}$.

\begin{tabular}{|c|c|c|c|}
\hline Model parameter, $\hat{\mathcal{A}}^{-1}$ & 0.3 & 0.5 & 0.7 \\
\hline \multicolumn{4}{|l|}{ Corrections due to differential rotation } \\
\hline Gravitational mass, $\delta M_{\mathrm{DR}}$ & $4.500(+31)$ & $1.335(+32)$ & $2.868(+32)$ \\
\hline Baryonic mass, $\delta M_{\mathrm{BDR}}$ & $6.495(+31)$ & $1.620(+32)$ & $3.296(+32)$ \\
\hline Proper mass, $\delta M_{\mathrm{PDR}}$ & $6.808(+31)$ & $1.704(+32)$ & $3.476(+32)$ \\
\hline \multicolumn{4}{|l|}{ Total quantities and significant ratios } \\
\hline Angular velocity ratio, $\Omega_{\mathrm{c}} / \Omega_{\mathrm{e}}$ & $1.131(+00)$ & $1.363(+00)$ & $1.712(+00)$ \\
\hline Gravitational mass, $M_{\text {tot }}$ & $2.206(+33)$ & $2.294(+33)$ & $2.447(+33)$ \\
\hline Baryonic mass, $M_{\text {Btot }}$ & $2.377(+33)$ & $2.474(+33)$ & $2.642(+33)$ \\
\hline Ratio $\left(\delta M_{\mathrm{B}}+\delta M_{\mathrm{BDR}}\right) / M_{\mathrm{B}}$ & $1.752(-01)$ & $2.230(-01)$ & $3.058(-01)$ \\
\hline Proper mass, $M_{\text {Ptot }}$ & $2.484(+33)$ & $2.586(+33)$ & $2.763(+33)$ \\
\hline Angular momentum, $J_{\text {tot }}$ & $6.646(+48)$ & $7.217(+33)$ & $8.073(+48)$ \\
\hline Rotational kinetic energy, $T_{\text {tot }}$ & $2.266(+52)$ & $2.691(+52)$ & $3.426(+52)$ \\
\hline Gravitational potential energy, $|W|_{\text {tot }}$ & $2.726(+53)$ & $2.892(+53)$ & $3.181(+53)$ \\
\hline Ratio $(T /|W|)_{\text {tot }}$ & $8.315(-02)$ & $9.306(-02)$ & $1.077(-01)$ \\
\hline
\end{tabular}


Table 5. Numerical results given in cgs for the EOS FPS; differential rotation for three values of the model parameter $\hat{\mathcal{A}}^{-1}$.

\begin{tabular}{llll}
\hline Model parameter, $\hat{\mathcal{A}}^{-1}$ & \multicolumn{1}{c}{0.3} & 0.5 & 0.7 \\
\hline Corrections due to differential rotation & & & \\
Gravitational mass, $\delta M_{\mathrm{DR}}$ & $5.919(+31)$ & $1.759(+32)$ & $3.785(+32)$ \\
Baryonic mass, $\delta M_{\mathrm{BDR}}$ & $9.040(+31)$ & $2.212(+32)$ & $4.475(+32)$ \\
Proper mass, $\delta M_{\mathrm{PDR}}$ & $9.560(+31)$ & $2.351(+32)$ & $4.770(+32)$ \\
Total quantities and significant ratios & & & $1.778(+00)$ \\
Angular velocity ratio, $\Omega_{\mathrm{c}} / \Omega_{\mathrm{c}}$ & $1.143(+00)$ & $1.397(+00)$ & $2.943(+33)$ \\
Gravitational mass, $M_{\mathrm{tot}}$ & $2.624(+33)$ & $2.740(+33)$ & $3.236(+33)$ \\
Baryonic mass, $M_{\mathrm{Btot}}$ & $2.879(+33)$ & $3.010(+33)$ & $3.314(-01)$ \\
Ratio $\left(\delta M_{\mathrm{B}}+\delta M_{\mathrm{BDR}}\right) / M_{\mathrm{B}}$ & $1.862(-01)$ & $2.383(-01)$ & $3.404(+33)$ \\
Proper mass, $M_{\mathrm{Ptot}}$ & $3.022(+33)$ & $3.162(+33)$ & $1.168(+49)$ \\
Angular momentum, $J_{\mathrm{tot}}$ & $9.516(+48)$ & $1.038(+49)$ & $5.524(+52)$ \\
Rotational kinetic energy, $J_{\mathrm{tot}}$ & $3.568(+52)$ & $4.281(+52)$ & $4.216(+53)$ \\
Gravitational potential energy, $|W|_{\text {tot }}$ & $3.940(+53)$ & $1.015(-01)$ & $1.177(-01)$ \\
Ratio $(T / W \mid)_{\text {tot }}$ & $9.056(-02)$ & & $4.693(+53)$ \\
\hline
\end{tabular}

Table 6. Numerical results given in cgs for the EOS G; differential rotation for three values of the model parameter $\hat{\mathcal{A}}^{-1}$.

\begin{tabular}{llll}
\hline Model parameter, $\hat{\mathcal{A}}^{-1}$ & 0.3 & 0.5 & 0.7 \\
\hline Corrections due to differential rotation & & & \\
Gravitational mass, $\delta M_{\mathrm{DR}}$ & $1.769(+31)$ & $5.268(+31)$ & $1.137(+32)$ \\
Baryonic mass, $\delta M_{\mathrm{BDR}}$ & $2.294(+31)$ & $6.089(+31)$ & $1.270(+32)$ \\
Proper mass, $\delta M_{\mathrm{PDR}}$ & $2.323(+31)$ & $6.157(+31)$ & $1.284(+32)$ \\
Total quantities and significant ratios & & & $1.600(+00)$ \\
Angular velocity ratio, $\Omega_{\mathrm{c}} / \Omega_{\mathrm{c}}$ & $1.110(+00)$ & $1.306(+00)$ & $1.239(+33)$ \\
Gravitational mass, $M_{\mathrm{tot}}$ & $1.143(+33)$ & $1.178(+33)$ & $1.296(+33)$ \\
Baryonic mass, $M_{\mathrm{Btot}}$ & $1.192(+33)$ & $1.230(+33)$ & $2.421(-01)$ \\
Ratio $\left(\delta M_{\mathrm{B}}+\delta M_{\mathrm{BDR}}\right) / M_{\mathrm{B}}$ & $1.424(-01)$ & $1.787(-01)$ & $1.335(+33)$ \\
Proper mass, $M_{\mathrm{P} \text { tot }}$ & $1.390(+33)$ & $1.269(+33)$ & $2.102(+48)$ \\
Angular momentum, $J_{\text {tot }}$ & $1.750(+48)$ & $1.891(+48)$ & $7.399(+51)$ \\
Rotational kinetic energy, $T_{\text {tot }}$ & $5.042(+51)$ & $5.915(+51)$ & $9.392(+52)$ \\
Gravitational potential energy, $|W|_{\text {tot }}$ & $8.328(+52)$ & $8.716(+52)$ & $7.878(-02)$ \\
Ratio $(T /|W|)_{\text {tot }}$ & $6.054(-02)$ & $6.785(-02)$ & \\
\hline
\end{tabular}

owing to differential rotation, we find that the ratio $\left(\delta M_{\mathrm{B}}+\delta M_{\mathrm{BDR}}\right) / M_{\mathrm{B}}$ takes its larger value for the EOS AU which is the most stiff among the EOSs examined. It is worth reminding here that, considering a group of EOSs, most stiff is the EOS yielding the larger pressure for a particular density, and most soft is the EOS yielding the smaller pressure for that density among the EOSs of the group. Note, in addition, that the EOSs AU, C, and FPS are compatible in their stiffness for the density
$1 \times 10^{15} \mathrm{~g} \cdot \mathrm{cm}^{-3}$, next is the EOS F and most soft in the group is the EOS G; for larger densities, AU clearly becomes the most stiff, $\mathrm{C}$ and FPS continue to be compatible, $F$ is the next one, and $G$ remains the most soft in the group. Sorting our EOSs this way, we find that the change in mass decreases with the stiffness of the EOSs.

The "equatorial differential rotation profiles" (EDRP) $\delta \Omega / \Omega_{\mathrm{K}}$ are shown for our models in Figures 1, 3, 5, 7, and 9. Finally, Figures 2, 4, 6, 8, and 10 show the 


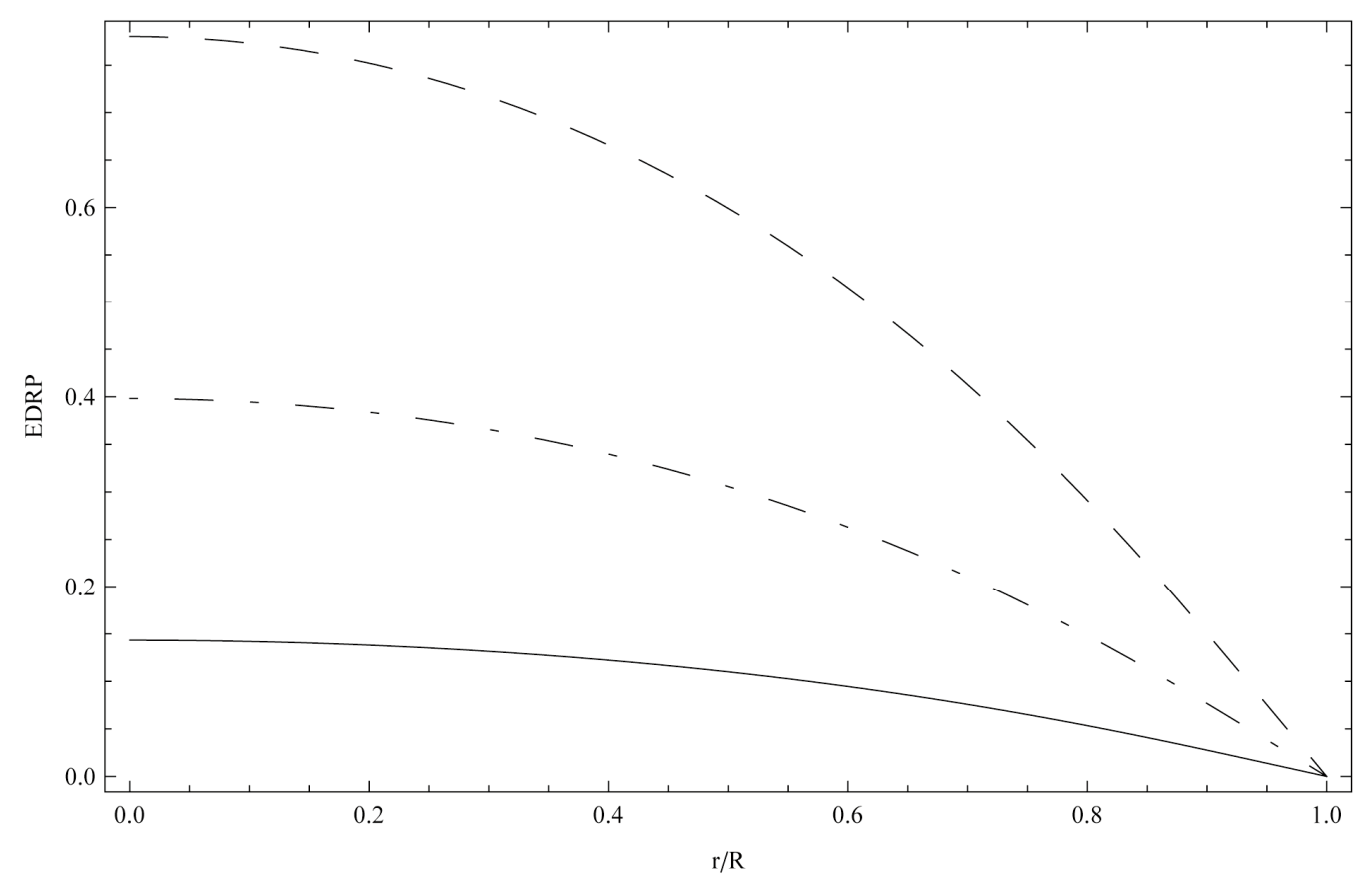

Figure 1. Equatorial differential rotation profile, EDRP $=\delta \Omega / \Omega_{\mathrm{K}}$, as a function of the ratio $r / R$ for the model obeying the EOS AU and for three values of the parameter $\hat{\mathcal{A}}^{-1}$ (solid line: $\hat{\mathcal{A}}^{-1}=0.3$, dotted-dashed line: $\hat{\mathcal{A}}^{-1}=0.5$, dashed line: $\left.\hat{\mathcal{A}}^{-1}=0.7\right)$.

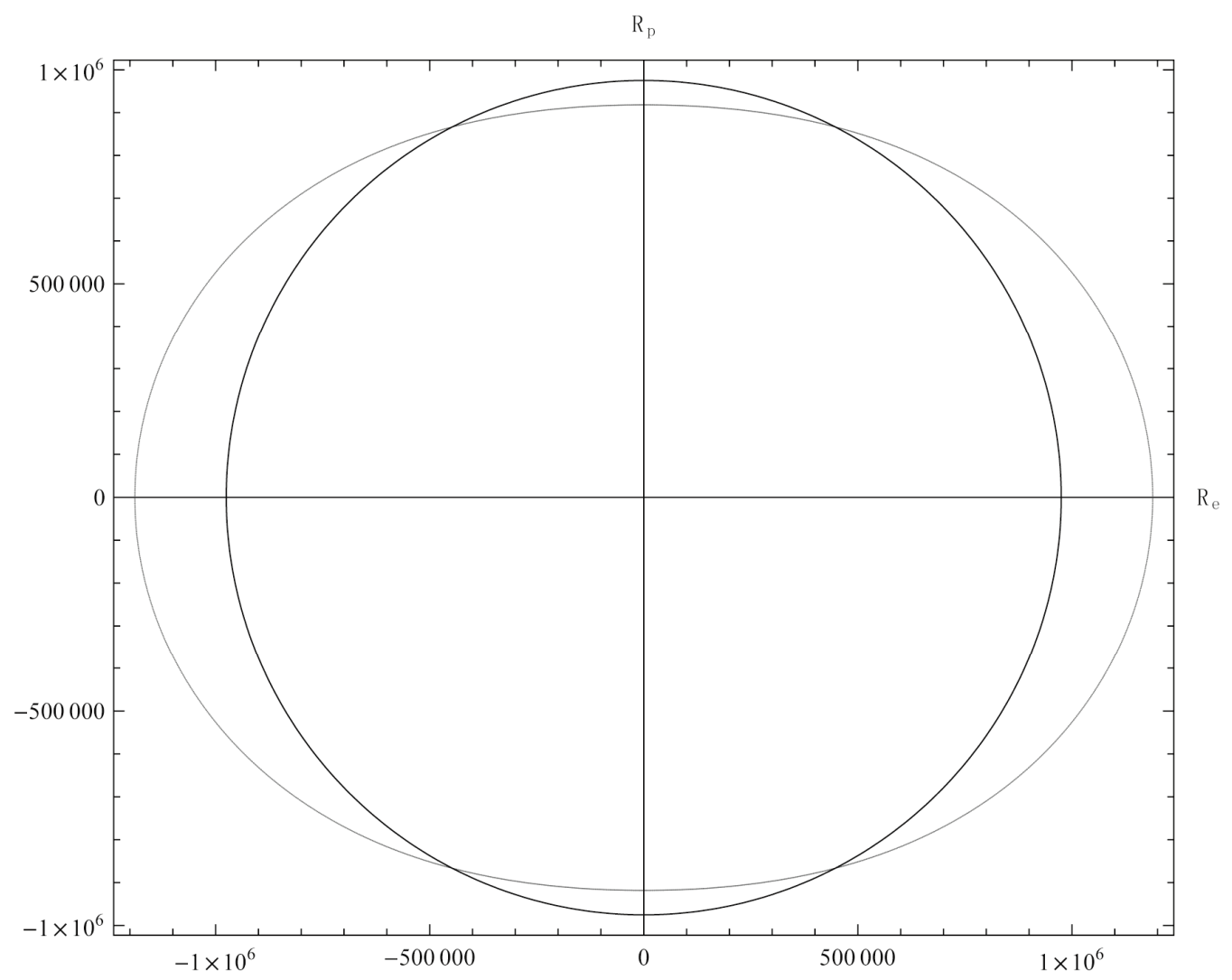

Figure 2. Meridional cross section for the model obeying the EOS AU and for the value $\hat{\mathcal{A}}^{-1}=0.7$ (bold line; soft line: the unperturbed model). 


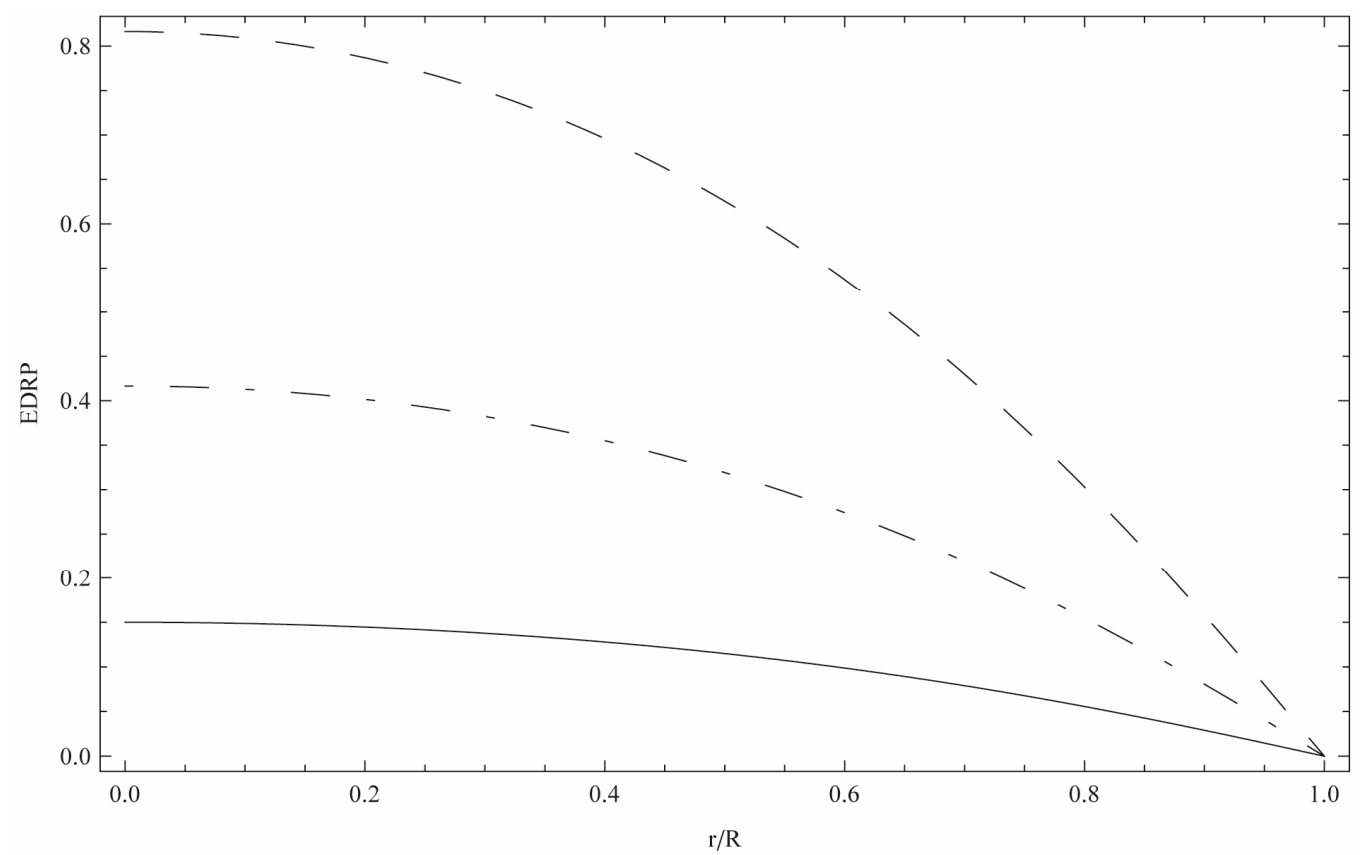

Figure 3. Equatorial differential rotation profile, EDRP $=\delta \Omega / \Omega_{\mathrm{K}}$, as a function of the ratio $r / R$ for the model obeying the EOS $C$ and for three values of the parameter $\hat{\mathcal{A}}^{-1}$ (solid line: $\hat{\mathcal{A}}^{-1}=0.3$, dotted-dashed line: $\hat{\mathcal{A}}^{-1}=0.5$, dashed line: $\left.\hat{\mathcal{A}}^{-1}=0.7\right)$.

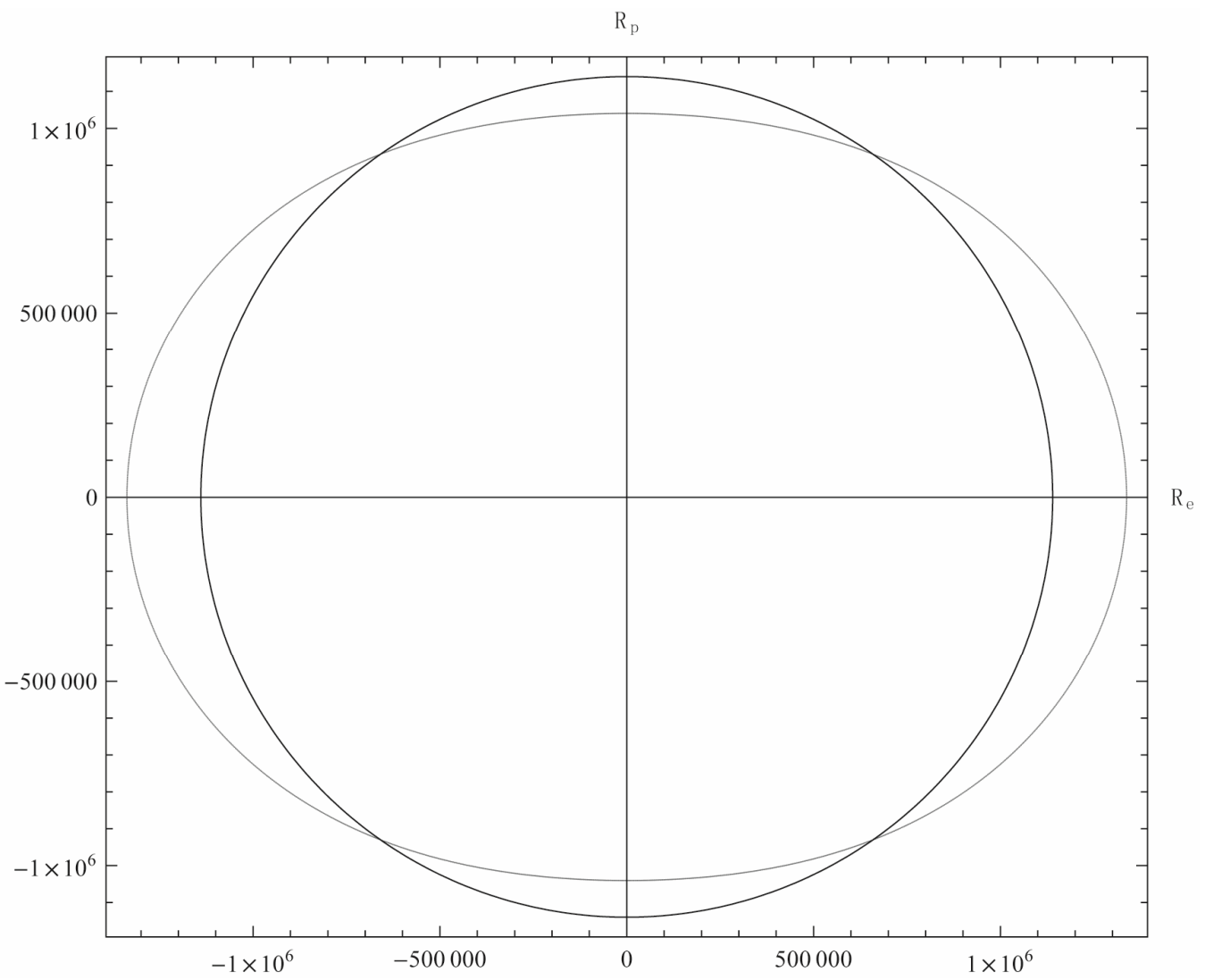

Figure 4. Meridional cross section for the model obeying the EOS C and for the value $\hat{\mathcal{A}}^{-1}=0.7$ (bold line; soft line: the unperturbed model). 


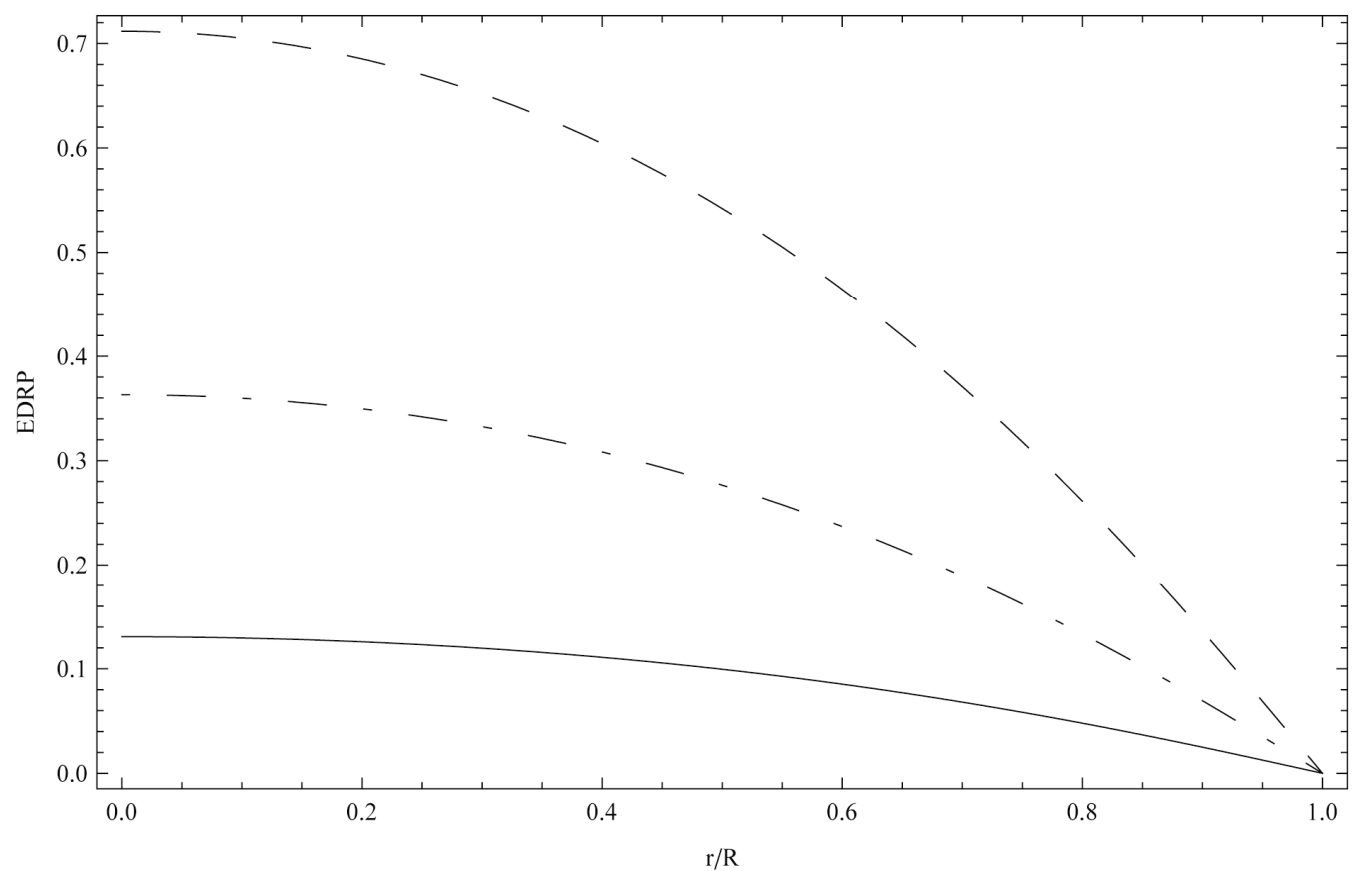

Figure 5. Equatorial differential rotation profile, EDRP $=\delta \Omega / \Omega_{\mathrm{K}}$, as a function of the ratio $r / R$ for the model obeying the EOS $F$ and for three values of the parameter $\hat{\mathcal{A}}^{-1}$ (solid line: $\hat{\mathcal{A}}^{-1}=0.3$, dotted-dashed line: $\hat{\mathcal{A}}^{-1}=0.5$, dashed line: $\left.\hat{\mathcal{A}}^{-1}=0.7\right)$.

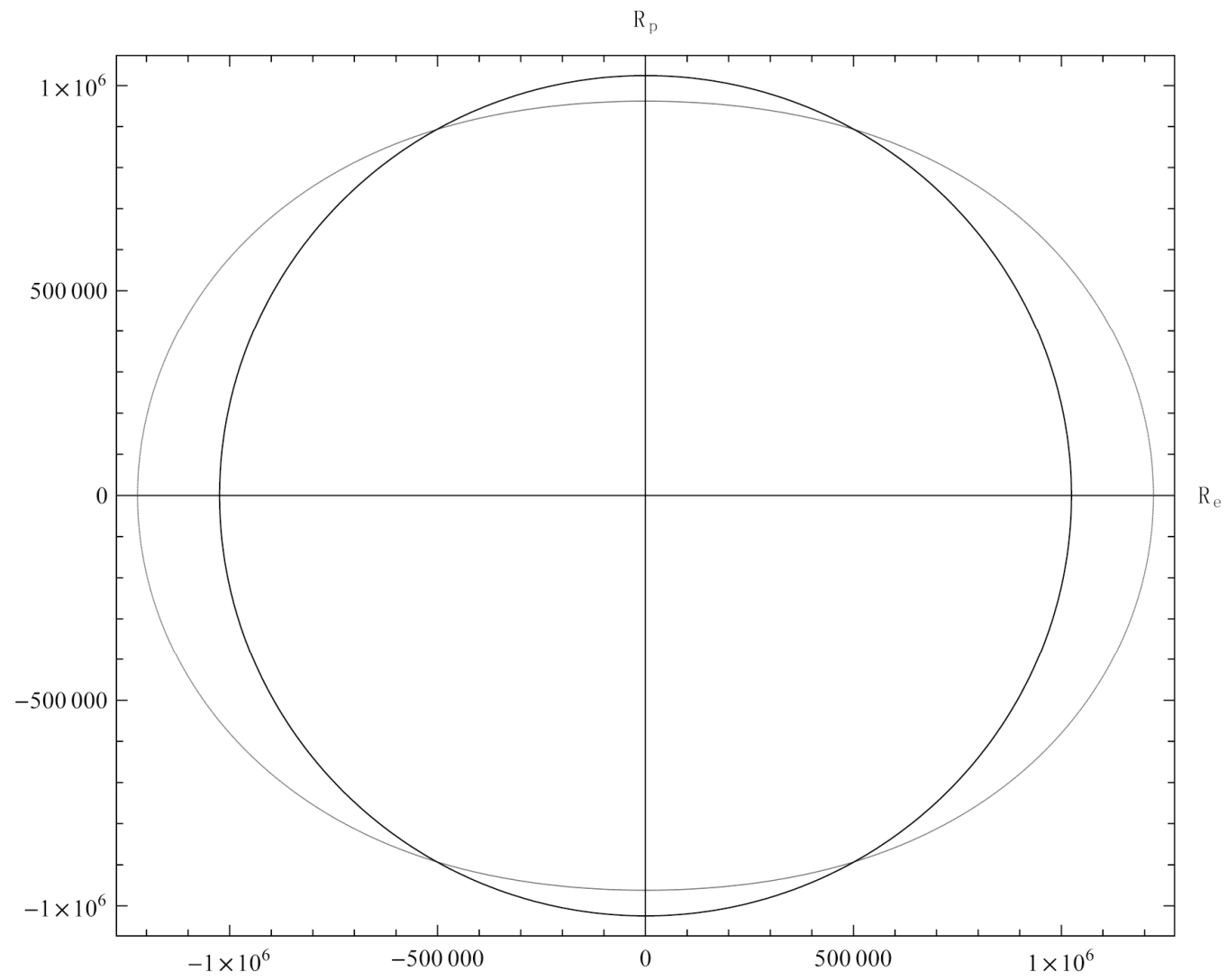

Figure 6. Meridional cross section for the model obeying the EOS F and for the value $\hat{\mathcal{A}}^{-1}=0.7$ (bold line; soft line: the unperturbed model). 


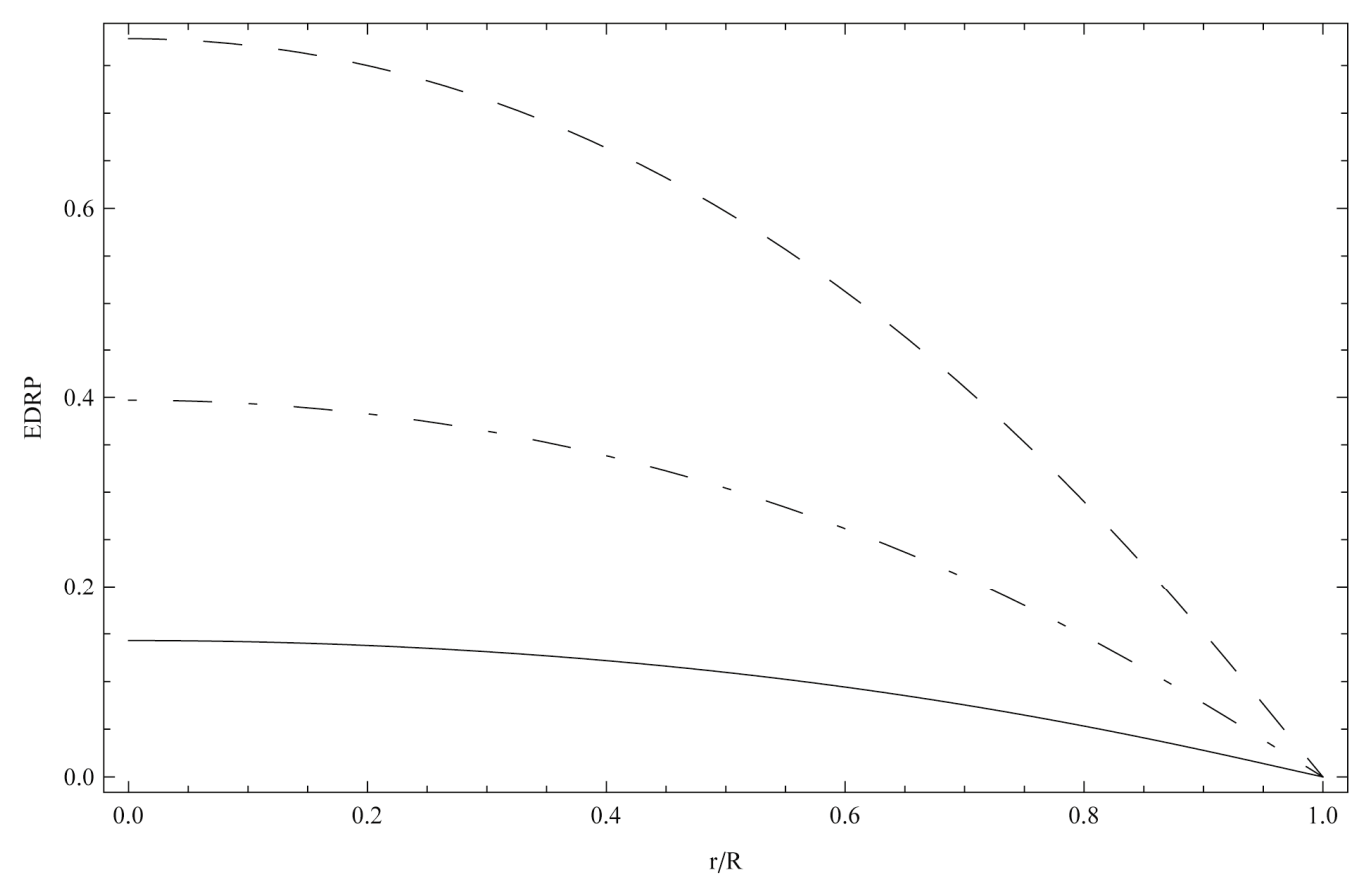

Figure 7. Equatorial differential rotation profile, EDRP $=\delta \Omega / \Omega_{\mathrm{K}}$, as a function of the ratio $r / R$ for the model obeying the EOS FPS and for three values of the parameter $\hat{\mathcal{A}}^{-1}$ (solid line: $\hat{\mathcal{A}}^{-1}=0.3$, dotted-dashed line: $\hat{\mathcal{A}}^{-1}=0.5$, dashed line: $\left.\hat{\mathcal{A}}^{-1}=0.7\right)$.

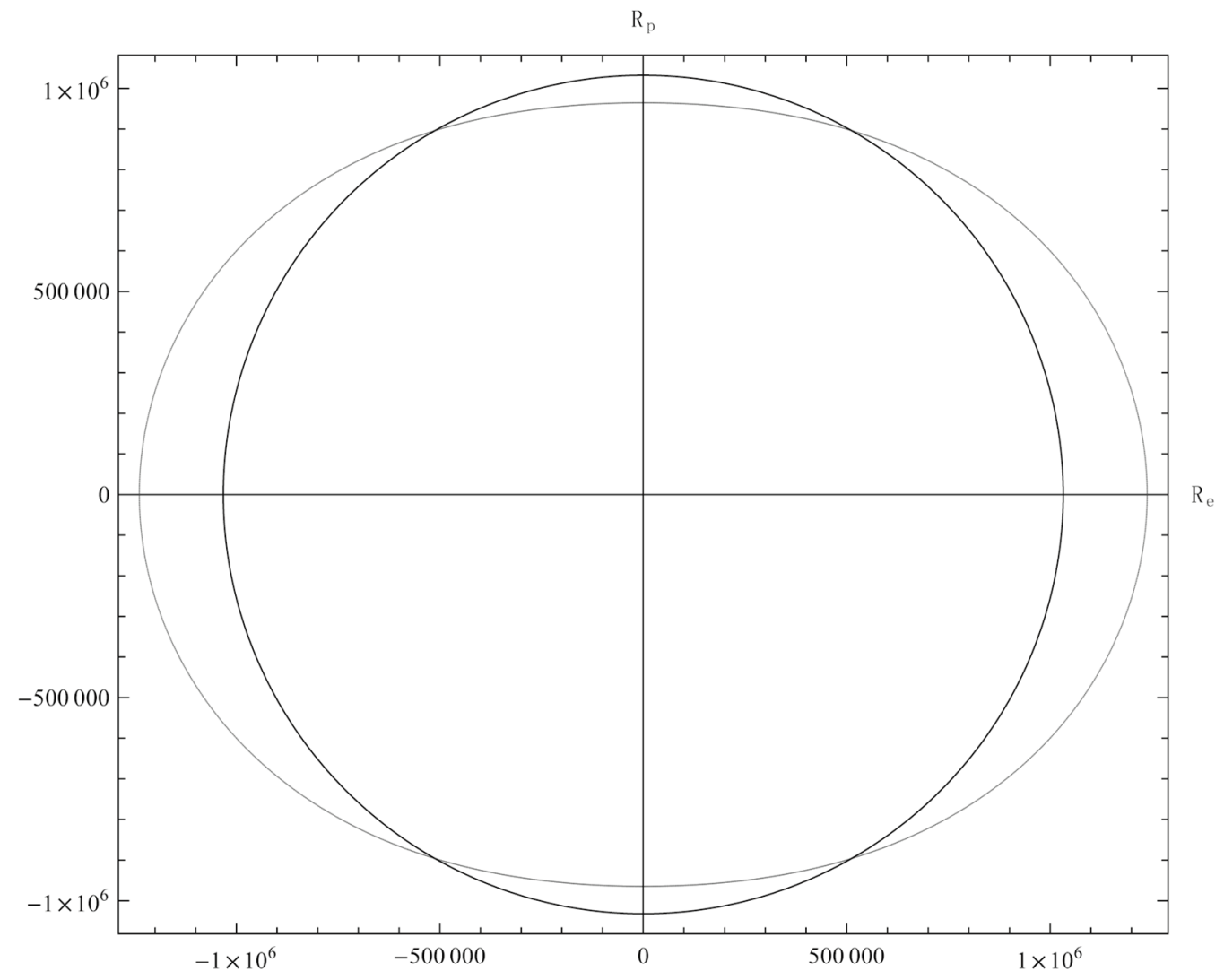

Figure 8. Meridional cross section for the model obeying the EOS FPS and for the value $\hat{\mathcal{A}}^{-1}=0.7$ (bold line; soft line: the unperturbed model). 


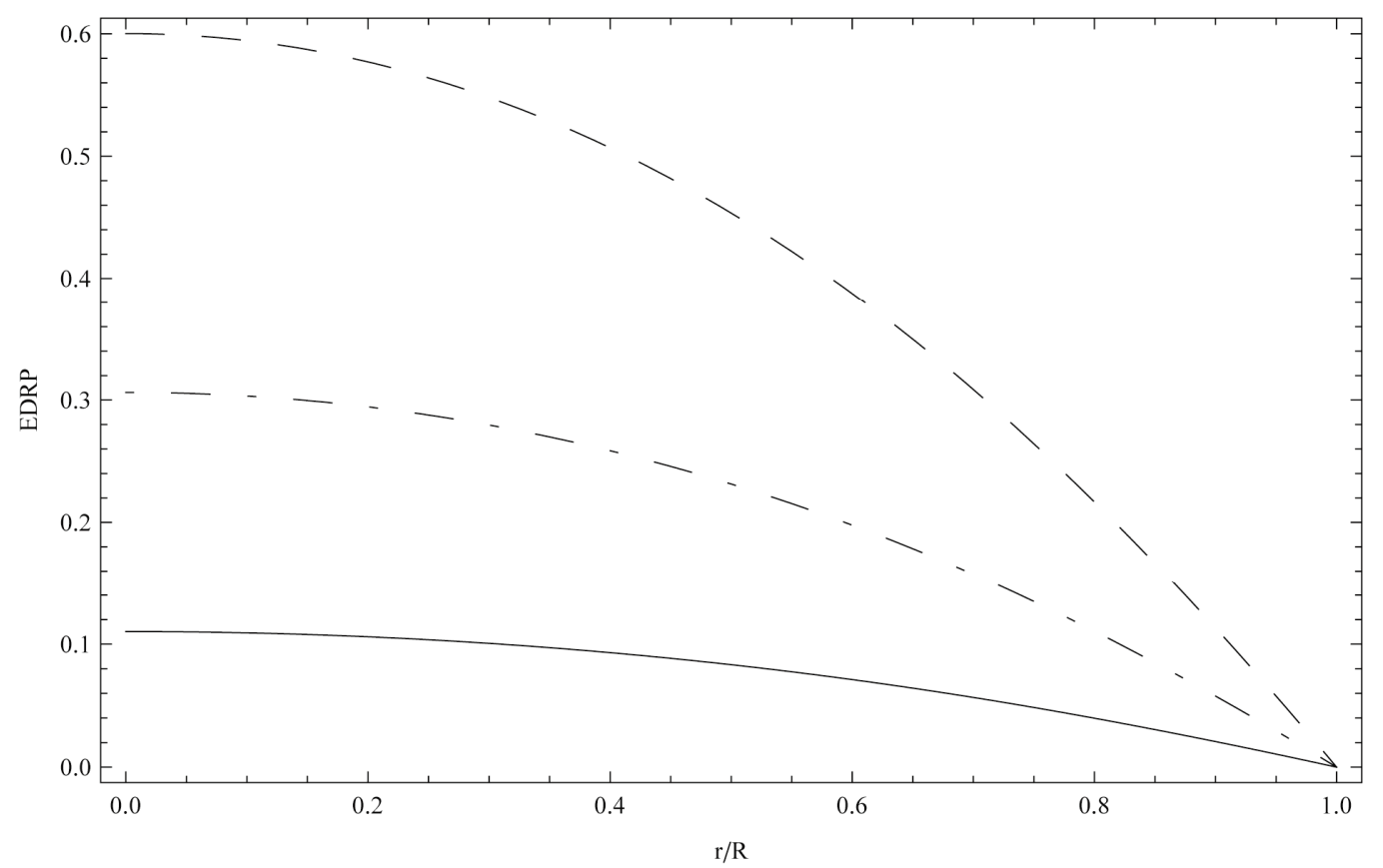

Figure 9. Equatorial differential rotation profile, EDRP $=\delta \Omega / \Omega_{\mathrm{K}}$, as a function of the ratio $r / R$ for the model obeying the EOS $G$ and for three values of the parameter $\hat{\mathcal{A}}^{-1}$ (solid line: $\hat{\mathcal{A}}^{-1}=0.3$, dotted-dashed line: $\hat{\mathcal{A}}^{-1}=0.5$, dashed line: $\hat{\mathcal{A}}^{-1}=0.7$ ).

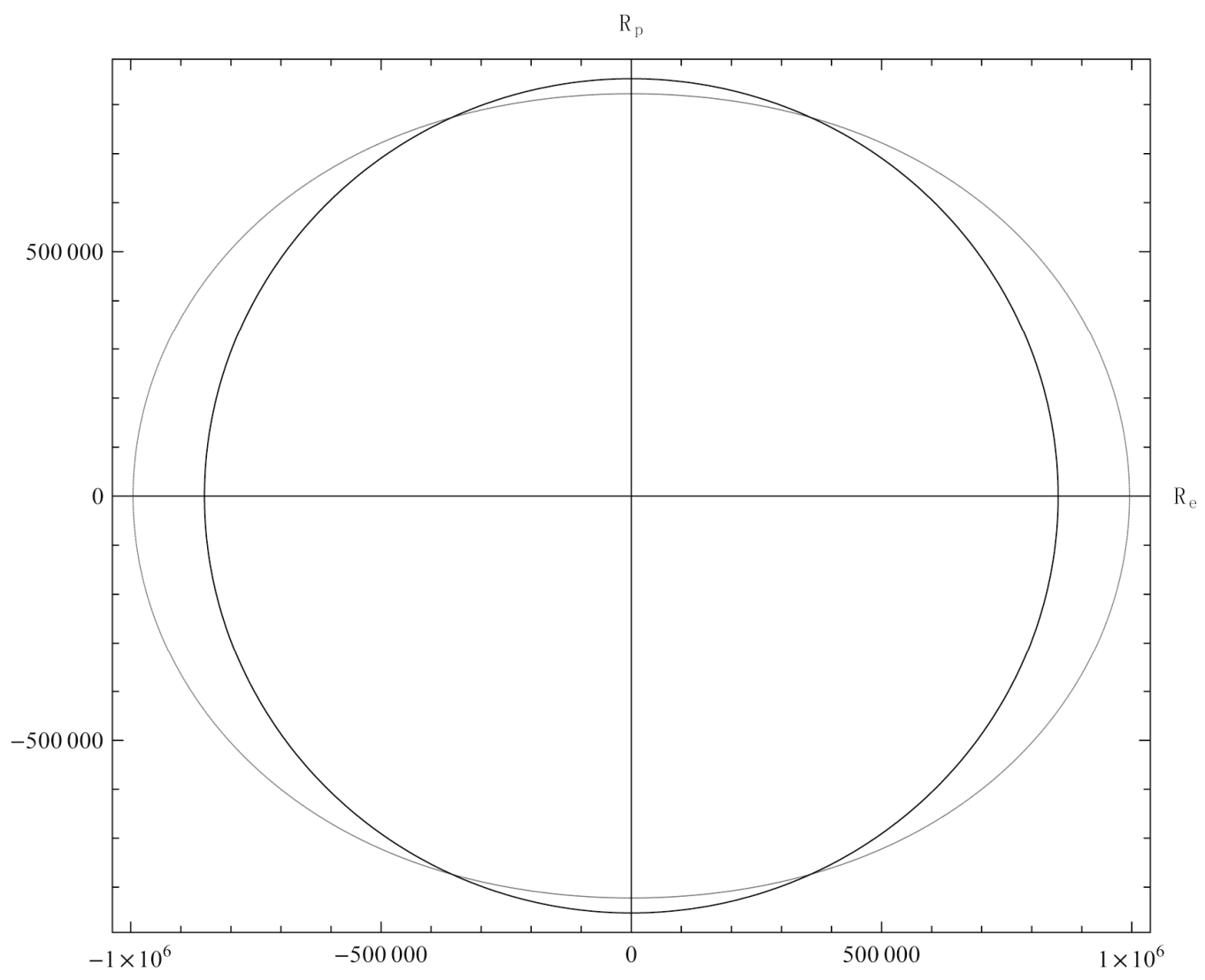

Figure 10. Meridional cross section for the model obeying the EOS G and for the value $\hat{\mathcal{A}}^{-1}=0.7$ (bold line; soft line: the unperturbed model). 
"meridional cross sections" of our models when the parameter $\hat{\mathcal{A}}^{-1}$ takes its larger value studied here, i.e. $\hat{\mathcal{A}}^{-1}=0.7$.

\section{REFERENCES}

[1] V. S. Geroyannis and A. G. Katelouzos, "Numerical Treatment of Hartle's Perturbation Method for Differentially Rotating Neutron Stars Simulated by GeneralRelativistic Polytropic Models," International Journal of Modern Physics C, Vol. 19, No. 12, 2008, pp. 1863-1908. doi:10.1142/S0129183108013370

[2] J. B. Hartle, "Slowly Rotating Relativistic Stars-I. Equations of Structure," The Astrophysical Journal, Vol. 150, 1967, pp. 1005-1029. doi:10.1086/149400

[3] J. B. Hartle and K. S. Thorne, "Slowly Rotating Relativistic Stars-II. Models for Neutron Stars and Supemassive Stars," The Astrophysical Journal, Vol. 153, 1968, pp. 807-834. doi:10.1086/149707

[4] J. B. Hartle, "Slowly Rotating Relativistic Stars-IX. Moments of Inertia of Rotationally Distorted Stars," Astrophysics and Space Science, Vol. 24, No. 2, 1973, pp. 385405. doi:10.1007/BF02637163

[5] I. A. Morrison, T. W. Baumgarte and S. L. Shapiro, "Effect of Differential Rotation on the Maximum Mass of Neutron Stars: Realistic Nuclear Equations of State," The Astrophysical Journal, Vol. 610, No. 2, 2004, pp. 941947. doi: $10.1086 / 421897$

[6] V. Pandharipande, "Dense Neutron Matter with Realistic Interaction," Nuclear Physics A, Vol. 174, No. 3, 1971, pp. 641-656. doi:10.1016/0375-9474(71)90413-1

[7] J. Arponen, "Internal Structure of Neutron Stars," $\mathrm{Nu}$ - clear Physics A, Vol. 191, No. 2, 1972, pp. 257-282. doi:10.1016/0375-9474(72)90515-5

[8] V. Canuto and S. M. Chitre, "Cristallization of Dense NeuTron Stars," Physical Review D, Vol. 9, No. 6, 1974, pp. 1587-1613. doi:10.1103/PhysRevD.9.1587

[9] A. Fabrocini, V. Fiks and R. B. Wiringa, "Equation of State for Dense Nucleon Matter," Physical Review C, Vol. 38, No. 2, 1988, pp. 1010-1037. doi:10.1103/PhysRevC.38.1010

[10] J. W. Negele and D. Vautherin, "Neutron Star Matter at Sub-Nuclear Densities," Nuclear Physics A, Vol. 207, No. 2, 1973, pp. 298-320. doi:10.1016/0375-9474(73)90349-7

[11] R. P. Feynman, N. Metropolis and E. Teller, "Equation of State of Elements Based on the Generalized Fermi-Thomas Theory," Physical Review, Vol. 75, No. 10, 1949, pp. 1561-1573. doi:10.1103/PhysRev.75.1561

[12] G. Baym, C. Pethick and P. Sutherland, "The Ground State of Matter at High Densities: Equation of State and Stellar Modles," The Astrophysical Journal, Vol. 170, 1971, pp. 299-317. doi:10.1086/151216

[13] G. Baym, H. A. Bethe and C. J. Pethick, "Neutron Star Matter," Nuclear Physics A, Vol. 175, No. 2, 1971, pp. 225-271. doi:10.1016/0375-9474(71)90281-8

[14] P. J. Papasotiriou and V. S. Geroyannis, "A SCILAB Program for Computing General-Relativistic Models of RoTating Neutron Stars by Implementing Hartle's Perturbation Method," International Journal of Modern Physics C, Vol. 14, No. 3, 2003, pp. 321-350. doi:10.1142/S0129183103004516

[15] N. Stergioulas, "Rotating Neutron Stars (RNS) Package," 1992. http://www.gravity.phys.uwm.edu/rns/index.html 